\title{
Observation of Substitutional Site Preference in a Quasicrystal and Implication on Local Structure
}

\author{
M. Eibschütz, M. E. Lines, H. S. Chen, and J. V. Waszczak \\ AT\& T Bell Laboratories, Murray Hill, New Jersey 07974 \\ and \\ G. Papaefthymiou and R. B. Frankel \\ Francis Bitter National Magnet Laboratory, Massachusetts Institute of Technology, Cambridge, Massachusetts 02139 \\ (Received 27 August 1987)
}

\begin{abstract}
A combination of magnetic susceptibility and Mössbauer measurements on quasicrystalline $i$ $\mathrm{Al}_{74} \mathrm{Mn}_{20-x} \mathrm{Fe}_{x} \mathrm{Si}_{6}(0.02 \leq x \leq 7.5)$ establishes that $\mathrm{Mn}$ atoms in $i-\mathrm{Al}_{74} \mathrm{Mn}_{20} \mathrm{Si}_{6}$ occupy two distinct classes of sites, and that $\mathrm{Fe}$ substitutes for only one of them. The two classes are distinguished by the possession or otherwise of a localized magnetic moment. The data are consistent with a structure of interconnecting Mackay icosahedra (MI) in which localized moments are possessed only by Mn atoms adjacent to "broken" MI connections. The implied connectivity of the resulting MI network is close to that anticipated for a packing of MI on a three-dimensional Penrose-tile lattice.

PACS numbers: 61.50.Em, 61.55.Dc, 76.80.+y
\end{abstract}

There has been intense experimental and theoretical interest in icosahedral (or " $i$-phase") quasicrystals ever since their discovery ${ }^{1}$ in 1984 . The bulk of the literature has focused on AlMn and AlMnSi alloys for which there is now a growing consensus ${ }^{2}$ that the prototypical "building block" is the so-called Mackay icosahedron (MI), ${ }^{3}$ formula unit $\mathrm{Mn}_{12}(\mathrm{Al}, \mathrm{Si})_{42}$, which is found in the crystalline ternary alloy $\alpha-(\mathrm{AlMnSi}){ }^{4}$ This MI consists of a vacant-centered icosahedron of twelve (defined as $\alpha$ site) $\mathrm{Al}, \mathrm{Si}$ inside an equioriented icosahedron of $\mathrm{Mn}$, with the remaining thirty ( $\beta$ site) Al,Si located at the midpoints of the edges of the $\mathrm{Mn}$ icosahedron. No differentiation between $\mathrm{Al}$ and $\mathrm{Si}$ location (when the latter is present) has yet been made.

The main uncertainty about these quasicrystals concerns the manner in which orientation-maintaining MI interconnect. In the crystalline $\alpha$ phase, they connect via additional ( $\gamma$ and $\delta$ site ${ }^{5}$ ) "glue" $\mathrm{Al}$ (or $\mathrm{Al}, \mathrm{Si}$ ) atoms along all eight $\langle 111\rangle$ directions of the bcc lattice upon which the MI centers are located, with no Mn atom being shared between adjacent MI. Extended x-rayabsorption fine-structure experiments ${ }^{6,7}$ clearly establish that the MI remain practically unchanged on passage to the quasicrystalline $i$ phase, although their connectivity via the glue atoms is significantly modified. Since the MI remain orientationally constrained and noncontacting in the $i$ phase, it seems clear that some of the $\alpha$-phase glue atom "bonds" must be "broken" in some sense on passage to the quasicrystal. Two kinds of models have been suggested, both involving MI connections along threefold icosahedral directions. One ${ }^{5,8,9}$ places the $i$ phase MI centers on lattice sites of a twelvefold-vertex ${ }^{5}$ three-dimensional Penrose-tile (3D-PT) lattice, while the other ${ }^{6,7,10,11}$ is based on a random-packing procedure.

Neither of these models contains within it any precise dual classification of inequivalent $\mathrm{Mn}$ (or $\mathrm{Al}, \mathrm{Si}$ ) sites, much less in any ratio involving the golden mean (since a formal decoration of $\mathrm{MI}$ in terms of Penrose tiles ${ }^{12}$ places most of the atoms on shared tiles). Nevertheless, a recent combination of NMR spin-echo ${ }^{13}$ and magnetic-susceptibility ${ }^{14}$ experiments has been interpreted in a manner which suggests that two distint classes of $\mathrm{Mn}$ sites can be distinguished both in AlMn and AlMnSi quasicrystals. They are those associated with the possession or not of a localized magnetic moment. Although some skepticism has been voiced ${ }^{15}$ concerning this interpretation, we shall establish unequivocally in this Letter that such a class separation of $\mathrm{Mn}$ sites does exist, and we shall offer a model of MI connectivity which rationalizes it.

Experimentally, we establish for the first time that $\mathrm{Fe}$ atoms substituting for $\mathrm{Mn}$ in $i-\mathrm{Al}_{74}\left(\mathrm{Mn}_{20-x} \mathrm{Fe}_{x}\right) \mathrm{Si}_{6}$ do not possess local magnetic moments and also do not influence the total paramagnetic moment of the sample. If follows that both magnetic and nonmagnetic $\mathrm{Mn}$ atoms must be present in these $i-(\mathrm{AlMnSi})$ alloys and that $\mathrm{Fe}$ selectively substitutes only from among the latter. This is the first observation of substantional site preference in any quasicrystal.

Alloys of $\mathrm{Al}_{74} \mathrm{Mn}_{20-x} \mathrm{Fe}_{x} \mathrm{Si}_{6}(0 \leq x \leq 7.5)$ were prepared by induction melting of high-purity $\mathrm{Al}, \mathrm{Si}, \mathrm{Mn}$, and $\mathrm{Fe}$ in a boron nitride crucible under argon atmosphere. Ribbon samples of about $1-\mathrm{mm}$ width and $30-\mu \mathrm{m}$ thickness were obtained by melt spun on a copper wheel $\approx 20 \mathrm{~cm}$ in diameter rotating at $2000 \mathrm{rpm}$. X-ray diffraction measurements for $0 \leq x \leq 7.5$ confirm $100 \%$ $i$-phase structure. For $x>7.5$, an additional $\mathrm{x}$-ray line is observed, indicating a multiple-phase material. TEM examination of these alloys showed domains of uniform contrast with faceted boundaries as observed in Al-SiMn quasicrystals. ${ }^{16}$ The magnetic susceptibility was measured by the Faraday method from 4.2 to $300 \mathrm{~K}$. 


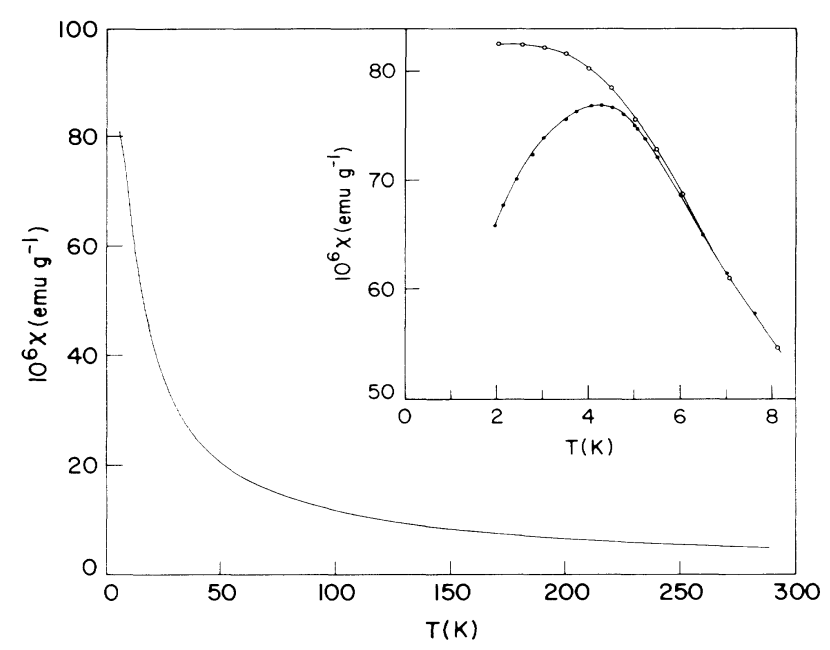

FIG. 1. Temperature dependence of magnetic susceptibility of the quasicrystal $i-\mathrm{Al}_{74} \mathrm{Mn}_{15} \mathrm{Fe}_{5} \mathrm{Si}_{6}$. Inset: Expanded plot of the low-temperature susceptibility for a sample initially cooled in zero field (filled circles) and one initially cooled in a magnetic field of 50 Oe (open circles).

The low-field susceptibility date measurements were made on a SHE Corp. SQUID magnetometer. The Mössbauer absorption spectra were obtained in a standard transmission geometry with a ${ }^{57} \mathrm{Co}$-in-Rh source.

The magnetic susceptibility $\chi$ as a function of temperature $T$ is qualitatively similar for all $i$-phase samples measured (Fig. 1). In particular, between 20 and $300 \mathrm{~K}$ it follows closely a Curie-Weiss dependence of the form $\chi=N p_{\text {eff }}^{2} / 3 k(T+\theta)+\chi_{0} \mathrm{emu} / \mathrm{g}$, where $N$ is the number of magnetic moments per gram, each of effective moment $p_{\text {eff }}=g \mu_{\mathrm{B}}[S(S+1)]^{1 / 2}$ (in conventional magnetic notation $\left.{ }^{17}\right), \theta$ is the Weiss temperature, and $\chi_{0}$ is a temperature-independent "Pauli" contribution from unfilled-band electrons. A typical experimental susceptibil-

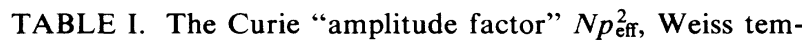
perature $\theta$, and Pauli paramagnetic component $\chi_{0}$ as deduced from measured magnetic susceptibility for quasicrystalline samples $i-\mathrm{Al}_{74} \mathrm{Mn}_{20-x} \mathrm{Fe}_{x} \mathrm{Si}_{6}$ by fitting with the Curie-Weiss equation between $T=20 \mathrm{~K}$ and room temperatures (see text). Also shown is the local magnetic moment $p_{\text {eff }}$ per magnetic $\mathrm{Mn}$ atom, and the peak-to-peak quadrupole splitting (QS) and isomer shift (IS) (with respect to iron metal at room temperature) as determined from the $T=4.2 \mathrm{~K}$ Mössbauer data.

\begin{tabular}{lcccccc}
\hline \hline & $\begin{array}{c}N p_{\text {eff }}^{2} \\
x\end{array}$ & $\begin{array}{c}\theta \\
\left(10^{21} \mu_{\mathrm{B}}^{2} / \mathrm{g}\right)\end{array}$ & $\begin{array}{c}\chi_{0} \\
(\mathrm{~K})\end{array}$ & $\begin{array}{c}p_{\text {eff }} \\
\left(10^{6} \mathrm{emu} / \mathrm{g}\right)\end{array}$ & $\begin{array}{c}\mathrm{QS} \\
\left(\mu_{\mathrm{B}}\right)\end{array}$ & $\begin{array}{c}\mathrm{IS} \\
(\mathrm{mm} / \mathrm{s})\end{array}$ \\
\hline 0.02 & 6.8 & 13 & 0.9 & 1.7 & 0.41 & 0.33 \\
2.5 & 6.2 & 10 & 0.6 & 1.6 & 0.43 & 0.33 \\
5.0 & 5.9 & 10 & 0.7 & 1.6 & 0.42 & 0.33 \\
7.0 & 6.5 & 10 & 0.5 & 1.7 & 0.41 & 0.33 \\
7.5 & 6.4 & 12 & 0.9 & 1.7 & 0.41 & 0.32 \\
\hline \hline
\end{tabular}

ity plot for $i-\mathrm{Al}_{74} \mathrm{Mn}_{15} \mathrm{Fe}_{5} \mathrm{Si}_{6}$ is shown in Fig. 1. Computer fitting with the above equation between 20 and 300 $\mathrm{K}$ for each iron concentration enables us to extract the relevant magnetic parameters $N p_{\text {eff }}^{2}, \theta$, and $\chi_{0}$ for each composition. They are shown in Table I.

We note from Table I that both $N p_{\text {eff }}^{2}$ and $\theta$ are essentially independent of iron concentration $x$ (the small variations as a function of $x$ being within the experimental scatter of the data). The sign of $\theta$ is antiferromagnetic, suggesting the possibility of spin-glass ordering at low temperatures. Indeed, detailed susceptibility measurements below $8 \mathrm{~K}$ (e.g., Fig. 1 inset) are typical of those expected for a spin-glass transition at or below about $4 \mathrm{~K}$. The constancy of $N p_{\text {eff }}^{2}$ as a function of $x$ implies that those iron atoms substituting for $\mathrm{Mn}$ in $i$ (AlMnSi) possess the same magnetic moment as do the Mn which they replace. The Mössbauer experiments described below establish beyond question that this moment is zero. It follows, since $p_{\text {eff }} \neq 0$, that there must be two separate classes of $\mathrm{Mn}$ in $i-(\mathrm{AlMnSi})$, one possessing a local magnetic moment and one not. For reasons not immediately apparent, iron is only able to substitute for the latter. If the limiting concentration $x=7.5$ of iron which will support the $i$ phase exhausts all of the "nonmagnetic" Mn sites (and we shall argue below that this is likely to be the case), then the number of $\mathrm{Mn}$ sites per gram of $i-\mathrm{Al}_{74} \mathrm{Mn}_{20} \mathrm{Si}_{6}$ which carry a localized magnetic moment is readily calculated as $N=12.5 N_{0} / M$, where $N_{0}=6 \times 10^{23}$ is Avogadro's number, and $M=3264$ is the mass (in atomic mass units) of the 100-atom formula unit. Coupling this value of $N$ with the findings for $N p_{\text {eff }}^{2}$ in Table I now enables us to deduce the effective magnetic moment $p_{\text {eff }} \approx(1.6-1.7) \mu_{\text {B }}$ per local moment (see Table I).

The room-temperature and 4.2-K zero-field Mössbauer quadrupole spectra for the $i$-phase compositions $\mathrm{Al}_{74} \mathrm{Mn}_{20-x} \mathrm{Fe}_{x} \mathrm{Si}_{6}$ are all closely similar in shape to that analyzed in detail for $i-\mathrm{Al}_{86}\left(\mathrm{Mn}_{0.98} \mathrm{Fe}_{0.02}\right)_{14}$ by Eibschütz, Chen, and Hauser ${ }^{18}$ and Swantzendrubber et al. ${ }^{18}$ An example, for $i-\mathrm{Al}_{74} \mathrm{Mn}_{15} \mathrm{Fe}_{5} \mathrm{Si}_{6}$ at $4.2 \mathrm{~K}$, is shown in Fig. 2(a). We record here (Table I) only the isomer shifts and peak-to-peak quadrupole splittings of the complete spectra, and note only that the isomer shifts are almost unchanged from the $\alpha$ phase of $\mathrm{AlMnSi}$, while the mean electric-field gradients are somewhat increased (by $40 \%-50 \%$ ) from the crystalline counterpart. $^{19}$

A most important experimental finding results from the high-field (80 kOe), low-temperature $(4.2 \mathrm{~K})$ Mössbauer spectra for the quasicrystalline samples, a typical example of which is shown in Fig. 2 (again for the composition $x=5$ ). The observed spectrum is exactly that expected ${ }^{20}$ for a ${ }^{57} \mathrm{Fe}$ nucleus interacting with an essentially sharp magnetic field of $80 \mathrm{kOe}$ parallel to the $\gamma$ ray and subject to a randomly oriented electric-field-gradient distribution of $\mathrm{rms}$ full width $\approx 0.5 \mathrm{~mm} / \mathrm{s}$, i.e., equal to 


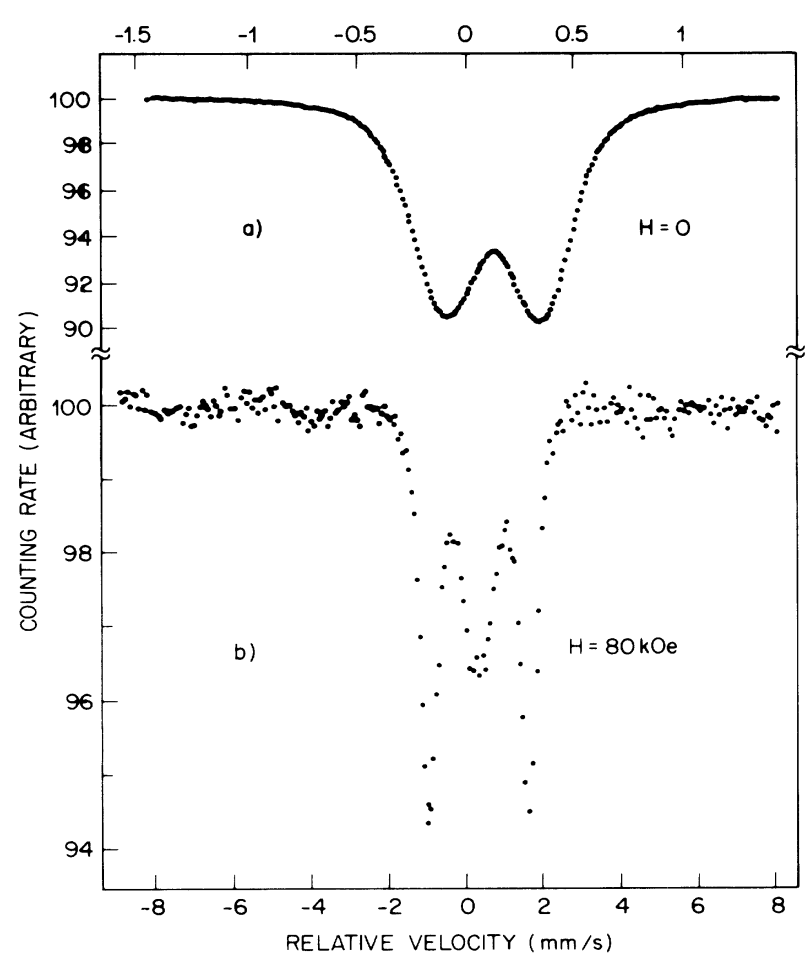

FIG. 2. (a) The $T=4.2 \mathrm{~K}$ Mössbauer spectrum of quasicrystalline $i$ - $\mathrm{Al}_{74} \mathrm{Mn}_{15} \mathrm{Fe}_{5} \mathrm{Si}_{6}$ and (b) the $T=4.2 \mathrm{~K}$ Mössbauer Zeeman spectrum of the same sample in a magnetic field $H=80 \mathrm{kOe}$ applied parallel to the direction of $\gamma$-ray propagation.

that of Fig. 2(a). The inference is that each iron nucleus experiences a total magnetic field essentially equal to the applied field alone-in other words, that the magnetically induced hyperfine field is negligible. This can only occur if the iron atoms carry no local moment. Any iron local moment (whether paramagnetic or incipiently spinglass ordered) in a field of $80 \mathrm{kOe}$ at $4.2 \mathrm{~K}$ would severely modify the Mössbauer Zeeman spectrum of Fig. 2(b).

Crystalline $\alpha-(\mathrm{AlMnSi})$ is, in essence, a bcc arrangement of MI, connected along (all eight) body-centered axes by octahedral chains of $\mathrm{Al}$ atoms. ${ }^{21}$ In detail, the origin and bcc-site MI are both slightly distorted from the prototypical configuration but in different ways, giving rise to two inequivalent $\mathrm{Mn}$ sites. ${ }^{4,13}$ However, both sites, with six (one $\alpha$ type, five $\beta$ type) Al,Si intra-MI nearest neighbors (nn) and either three or four ( $\gamma$ and $\delta$ type) Al,Si inter-MI glue nn, contribute a sufficient density of itinerant-electron states at a $\mathrm{Mn}$ site to suppress local moment formation. Crystalline $\alpha-(\mathrm{AlMnSi})$ is, consequently, a Pauli paramagnet. ${ }^{13,14}$

Since $\mathrm{Mn}$ is completely nonmagnetic ${ }^{19}$ (in the sense of not possessing a local moment) in the fully (eightfold) connected crystalline alloy, we propose that the nonmagnetic sites in the equivalent $i$-phase quasicrystalline alloy are those which are least perturbed on passage to the $i$ phase-namely those which are adjacent to retained threefold icosahedral $\alpha$-type MI connections. Within the same model it follows that the magnetic Mn sites in the $i$ phase must be those adjacent to "broken" MI connections. This picture receives support from the measured modulation of nn-pair $\mathrm{Mn}-\mathrm{Al}$ distributions ${ }^{22}$ on passage from the crystalline to the $i$ phase in both AlMn and AlMnSi alloys, ${ }^{23}$ for which the most perturbed bonds (i.e., those adjacent to broken MI connections) experience a significant increase in bond length. The resulting expanded Al "cage" would likely give rise to a smaller itinerant-electron density at these $\mathrm{Mn}$ sites and hence be more likely to favor conditions for the creation of a localized magnetic moment. ${ }^{24}$

Within our model we therefore identify two classes of $\mathrm{Mn}$ sites. The first is nonmagnetic, is more compact and less asymmetric as regards its $\mathrm{Al} \mathrm{nn}$ environment, and, being adjacent to "connected" MI, is only modestly perturbed from its crystalline "parent" site. The second supports a localized magnetic moment, is less compact and more asymmetric in $\mathrm{Al} \mathrm{nn}$ environment, is adjacent to a broken MI connection, and is very substantially perturbed from its crystalline parent site. This model is consistent with all the experimental findings presented if $\mathrm{Fe}$, which is smaller than $\mathrm{Mn}$, can only substitute for $\mathrm{Mn}$ in the smaller, less asymmetric class of sites. ${ }^{25}$

The literature ${ }^{26}$ indicates that the basic bcc $\alpha$ form of iron-doped $\alpha$-(AlMnSi) remains stable to high iron concentrations, Fe:Mn $\gtrsim 4: 1$, although the crystalline structure of pure $\alpha$-(AlFeSi) does constitute a different packing arrangement of MI. ${ }^{12,27}$ Since we find, via Mössbauer spectroscopy, ${ }^{19}$ that Fe can substitute for $\mathrm{Mn}$ in both symmetry sites in $\alpha$-(AlMnSi), we infer that it is highly likely that $\mathrm{Fe}$ can replace $\mathrm{Mn}$ in quasicrystalline $i$-(AlMnSi) in all nonmagnetic sites before destabilization of the structure. If this is so, then the maximum concentration of $\mathrm{Fe}$ which will substitute for $\mathrm{Mn}$ in $i$ (AlMnSi) without destabilization of the structure gives a measure of the connectivity (i.e., number of unbroken connections per MI) in the $i$ phase. The exact form of this relationship in a "random bond-breaking" approximation is easy to establish as follows.

A fully connected MI structure possesses eight $\langle 111\rangle-$ oriented "bonds" each involving three Mn. Thus, the removal of one bond affects three of the twelve MI Mn sites. Of the seven topologically distinct ways of removing two bonds, three will involve orientationally $\mathrm{nn}$ connections (affecting five $\mathrm{Mn}$ ) and four will involve independent connections (affecting six $\mathrm{Mn}$ ). If each is equally likely to occur, then the mean number of $\mathrm{Mn}$ which are rendered magnetic by the removal of two bonds per $\mathrm{MI}$ is $5\left(\frac{3}{7}\right)+6\left(\frac{4}{7}\right)=5.57$. The procedure is easily generalized to show that the mean number $N$ of Mn rendered magnetic per MI by the random removal of $n \alpha$-type bonds per MI is as follows: $N(n)=3(n=1)$, 5.57 (2), 7.71 (3), 9.43 (4), 10.71 (5), 11.57 (6), 12 (7), 


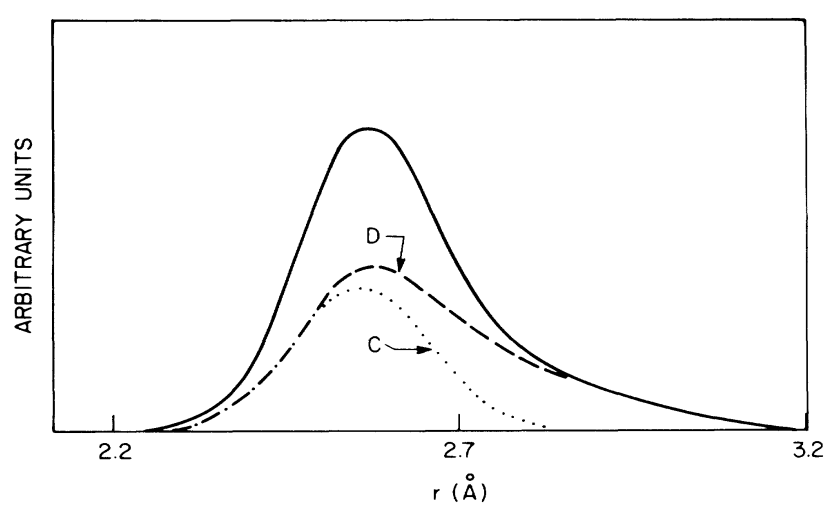

FIG. 3. The Mn-Al radial distribution function (full curve) of quasicrystalline $i-\mathrm{Al}_{84} \mathrm{Mn}_{16}$ as analyzed according to the model of the present Letter in terms of components corresponding to magnetic (curve $D$ ) and nonmagnetic (curve $C$ ) Mn atoms; see text.

\section{$12(8)$.}

Experimentally we find that the quasicrystalline $i$ phase in $\mathrm{Al}_{74} \mathrm{Mn}_{20-x} \mathrm{Fe}_{x} \mathrm{Si}_{6}$ can only be prepared for iron concentrations up to $x \approx 7.5$. This limiting concentration, interpreted as the filling of all nonmagnetic $\mathrm{Mn}$ sites by $\mathrm{Fe}$, implies that $12(7.5 / 20)=4.5 \mathrm{Mn}$ sites per MI do not support a local magnetic moment in $i$ $\mathrm{Al}_{74} \mathrm{Mn}_{20} \mathrm{Si}_{6}$ while $N=7.5$ do. From the implied function $N(n)$ we deduce a connectivity $c=8-n \approx 5.1$ in this quasicrystalline $i$ phase.

Previous estimates of MI connectivity have appeared in the literature for the $i$ phase. From an interpretation of extended x-ray-absorption fine-structure experiments, $\mathrm{Ma}$, Stern, and Bouldin ${ }^{7}$ deduce that $c$ is at least $\approx 3$ and elsewhere ${ }^{6}$ suggest (seemingly from that same data) that $c=3.4 \pm 1.0$. Theoretically, a model of dense randomly packed icosahedra sharing threefold faces is reported ${ }^{6}$ to have $c \approx 4$, while an icosahedrally decorated 3D-PT quasilattice of MI (with individual MI bridged without contact as in the real $i$ phase) has been prepared $^{8,9}$ with $c \approx 5.5$. The connectivity $c \approx 5.1$ resulting from our interpretation of the limiting Fe concentration seems therefore to be more in accord with the MI-decorated 3D-PT model than with one involving random icosahedral packing.

The model of this paper also suggests a reinterpretation of the $i$-phase $\mathrm{MnAl}$ distribution function of Ref. 23, Fig. 3. The computer decomposition of that figure into two symmetric Gaussians $A$ and $B$ (of respective areas $2.4: 1$ ) as given in Ref. 23 should, within the framework of the present model, now be reconstituted in the form $C=0.53 A$ and $D=0.47 A+B$ as depicted in Fig. 3. In the latter representation, curve $C$ corresponds to a fraction 7.5/20 of total $\mathrm{Mn}$ sites which are adjacent to unbroken MI connections and are correspondingly minimally perturbed from their crystalline distribution. Curve $D$ then establishes the somewhat more expanded distribution function relevant for the local-momentforming sites. Note that for the smaller radial values $r \lesssim 2.6 \AA$ (corresponding to intra-MI $\alpha$ and $\beta$ Al sites) curves $C$ and $D$ are of similar shape, as is required for physical self-consistency within the model.

The work by G. Papaefthymiou and R. B. Frankel at Francis Bitter National Magnet Laboratory was supported by the National Science Foundaton.

${ }^{1}$ D. Shechtman, I. Blech, D. Gratias, and J. W. Cahn, Phys. Rev. Lett. 53, 1951 (1984).

${ }^{2}$ C. L. Henley, Comments Condens. Matter Phys. 13, 59 (1987).

${ }^{3}$ A. L. Mackay, Acta Crystallogr. 15, 916 (1962).

${ }^{4} \mathrm{M}$. Cooper and K. Robinson, Acta Crystallogr. 20, 614 (1966).

${ }^{5}$ C. L. Henley, Phys. Rev. B 34, 797 (1986).

${ }^{6}$ E. A. Stern, Y. Ma, K. Bauer, and C. E. Bouldin, J. Phys. (Paris), Colloq. 47, C3-371 (1986).

${ }^{7}$ Y. Ma, E. A. Stern, and C. E. Bouldin, Phys. Rev. Lett. 57, 1611 (1986).

${ }^{8}$ P. Guyot, M. Audier, and R. Lequette, J. Phys. (Paris), Colloq. 47, C3-389 (1986)

${ }^{9}$ J. W. Cahn and D. Gratias, J. Phys. (Paris), Colloq. 47, C3-415 (1986).

${ }^{10}$ D. Schechtman and I. Blech, Metall. Trans. A 16, 1005 (1985).

${ }^{11}$ P. W. Stephens and A. I. Goldman, Phys. Rev. Lett. 56, 1168 (1986).

${ }^{12}$ V. Elser and C. L. Henley, Phys. Rev. Lett. 55, 2883 (1985).

${ }^{13}$ W. W. Warren, H. S. Chen, and G. P. Espinosa, Phys. Rev. B 34, 4902 (1986).

${ }^{14}$ J. J. Hauser, H. S. Chen, and J. V. Waszczak, Phys. Rev. B 33, 3577 (1986).

${ }^{15}$ L. H. Bennett, M. Rubinstein, G. Xiao, and C. L. Chen, J. Appl. Phys. 61, 4364 (1987).

${ }^{16}$ C. H. Chen and H. S. Chen, Phys. Rev. B 33, 2814 (1986).

${ }^{17}$ See, for example, D. C. Mattis, The Theory of Magnetism (Harper and Row, New York, 1965).

${ }_{18}$ M. Eibschütz, H. S. Chen, and J. J. Hauser, Phys. Rev. Lett. 56, 169 (1986); L. J. Swantzendrubber, D. Shechtman, L. Bendersky, and J. W. Cahn, Phys. Rev. B 32, 1383 (1985).

${ }^{19} \mathrm{M}$. Eibschütz, unpublished.

${ }^{20}$ M. E. Lines, J. Chem. Phys. 43, 721 (1982).

${ }^{21}$ P. Guyot and M. Audier, Philos. Mag. 52, L15 (1985).

${ }^{22} \mathrm{Here}$, and henceforth, an Al site implies $\mathrm{Al}, \mathrm{Si}$ in $\mathrm{Si}-$ containing alloys.

${ }^{23}$ J. B. Boyce, J. C. Mikkelsen, F. Bridges, and T. Egami, Phys. Rev. B 33, 7314 (1986).

${ }^{24}$ P. W. Anderson, Phys. Rev. 124, 41 (1971), and Science 201, 307 (1978).

${ }^{25} \mathrm{Mössbauer-effect} \mathrm{measurements} \mathrm{in} \mathrm{crystalline} \alpha$-AlMnSi confirm that $\mathrm{Fe}$ substitutes in both the origin and bcc MI Mn sites.

${ }^{26}$ M. Cooper, Acta Crystallogr. 23, 1106 (1967).

${ }^{27}$ R. N. Corby and P. J. Black, Acta Crsytallogr. Sect. B 33f, 3468 (1977). 